\title{
Fluorescence Detection 400-480 nm Using Microfluidic System Integrated GaP Photodiodes
}

\author{
Dion McIntosh, ${ }^{1}$ Qiugui Zhou, ${ }^{1}$ Francisco J. Lara, ${ }^{2}$ James Landers, ${ }^{2}$ and Joe C. Campbell \\ ${ }^{1}$ Department of Electrical Engineering, University of Virginia, 351 McCormick Road, Charlottesville, VA 22904, USA \\ ${ }^{2}$ Department of Chemistry, University of Virginia, McCormick Road, P.O. Box 400319, Charlottesville, VA 22904, USA
}

Correspondence should be addressed to Joe C. Campbell, jcc7s@virginia.edu

Received 29 April 2011; Revised 14 August 2011; Accepted 21 August 2011

Academic Editor: Zhihong Li

Copyright (C) 2011 Dion McIntosh et al. This is an open access article distributed under the Creative Commons Attribution License, which permits unrestricted use, distribution, and reproduction in any medium, provided the original work is properly cited.

\begin{abstract}
Ciprofloxacin is a commonly used antibiotic and the active ingredient in a veterinary antibiotic. Detecting its presence allows us to understand its absorption process in blood as well as tissue. A portable microfluidic system has been fabricated. It operates at low bias voltage and shows a linear relationship between concentration levels and system response. Detection of concentrations down to $1 \mathrm{ppb}$ of ciprofloxacin in microliters of solution was achieved.
\end{abstract}

\section{Introduction}

As the medical industry has evolved, it has become necessary to have accurate testing available in the shortest possible time [1-5]. Miniaturizing UV and fluorescence detectors and extending them to fluid analysis is one way of bridging this gap [6-9].

Detecting ciprofloxacin has become increasingly important because it is the active ingredient in enrofloxacin an antibiotic used in veterinary medicine $[10,11]$. Interest in monitoring ciprofloxacin levels in the tissues of animals raised for food and in the milk of cows has led to improvements in current technology and a search for new detection techniques. Ciprofloxacin absorbs strongly in the $\mathrm{UV}(\leq 280 \mathrm{~nm})$ and emits at $\sim 440 \mathrm{~nm}[10]$.

Currently, online detection is carried out by analytical methods such as high-performance liquid chromatography (HPLC), which involves a relatively costly investment in hardware $[12,13]$, or by capillary electrophoresis. Capillary electrophoresis (CE), while simpler than the aforementioned HPLC, still requires complex sample preparation methods utilizing $\mathrm{pH}$ adjustment to reach lower detection limits and requires very high bias voltage $(\mathrm{kV})[12,14]$. These two methods usually employ photomultiplier tubes (PMTs) for photodetection.

PMTs have been used in applications such as lowlevel ultraviolet detection in laser-induced fluorescence biological-agent warning systems [15]. Other applications in this wavelength range are under-water detection at $400 \mathrm{~nm}$, the wavelength at which water is transparent, and detection of $440 \mathrm{~nm}$-wavelength light from scintillation crystals that are used to sense gamma rays from nuclear material.

While PMTs are among the most sensitive detectors currently available, semiconductor photodiodes offer the advantages of being less expensive and more robust. Si photodiodes have high responsivities at $440 \mathrm{~nm}$; however, $\mathrm{GaP}$ exhibits a detection cutoff wavelength of $550 \mathrm{~nm}$, which makes it an attractive alternative to $\mathrm{Si}$, which requires expensive filters to reject extraneous wavelengths.

This paper expands on our previous success in integrating photodiodes with microfluidics for ciprofloxacin detection [16]. In that work, linear detection of $0.01 \mathrm{ppm}$ ciprofloxacin was achieved. The best published results for the widely used techniques are $0.01 \mathrm{ppm}$ HPLC and $0.015 \mathrm{ppm}$ CE $[12,14]$. We note however that these detection limits were obtained in physiological samples. It should also be pointed out that HPLC and CE provide richer spectral results than the technique reported here, which provides simple confirmation of the presence of ciprofloxacin.

\section{Fabrication}

$\mathrm{GaP}$ wafers were grown by metal organic chemical vapor deposition. The wafer structure was as follows: an n-type 


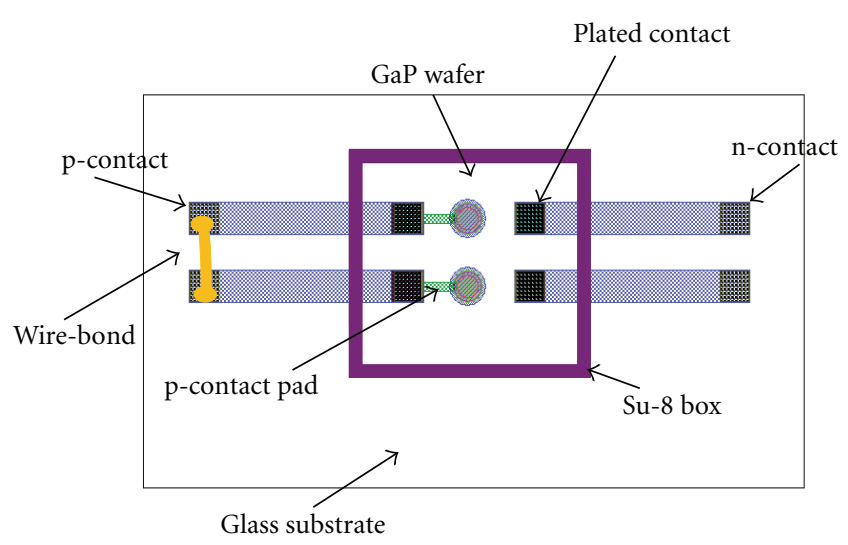

FIGURE 1: Detector system as viewed through the glass substrate.

substrate with a $650 \mathrm{~nm}$-thick $\mathrm{n}^{+}$-doped $\left(1.4 \times 10^{19} \mathrm{~cm}^{-3}\right)$ first layer, an $800 \mathrm{~nm}$-thick unintentionally undoped $(4 \times$ $\left.10^{16} \mathrm{~cm}^{-3}\right)$ second layer, and a $300 \mathrm{~nm} \mathrm{p}^{+}$-doped $(1.1 \times$ $10^{19} \mathrm{~cm}^{-3}$ ) top layer.

Mesa structure devices were fabricated by standard cleanroom processes. First, a mesa was defined by photolithography and etched to the bottom $\mathrm{n}^{+}$layer by inductively coupled plasma. This was followed by a 5 -second etch in $\mathrm{HNO}_{3}: \mathrm{HCl}:$ DI water in equal parts to remove damage to the sidewall caused by the inductively coupled plasma etch. Plasma enhanced chemical-vapor deposition was used to deposit $\mathrm{SiO}_{2}$ which served as both a passivation and an antireflection coating. After contacts were formed by metal evaporation of AuGe-Ni-Au $(40 \mathrm{~nm}, 10 \mathrm{~nm}, 110 \mathrm{~nm})$, the contacts were annealed for ten seconds at $430^{\circ} \mathrm{C}$ in a mixture of nitrogen and hydrogen [17]. A Ti-Au p-contact pad was evaporated and both the p-contact pad and $n$ contact were Au plated to a final thickness of $\sim 2 \mu \mathrm{m}$. The wafer was diced into $1 \mathrm{~mm}^{2}$ chips consisting of a $2 \times 1$ array of $\mathrm{GaP}$ photodiodes. In order to flip the contacts a corresponding metal contact $\mathrm{Ti}-\mathrm{Au}$ was evaporated on a thin glass slide. These contacts were Au plated at each end; the GaP photodiodes were bonded to the inner contacts and the outer contacts served as bias pads after flip-chip bonding. Using SU-8 photoresist, a box slightly larger than the chip and approximately $30 \mu \mathrm{m}$ in height was defined by photolithography. Its function was to facilitate alignment of the contacts on the $\mathrm{GaP}$ chips with those on the glass. The chips were bonded to the contact pads at $220^{\circ} \mathrm{C}$. The p-contact pads of two adjacent devices were wire bonded together placing the photodiodes in parallel with each other (see Figure 1).

\section{Device Characteristics}

The dark currents of the individual devices were 0.06 and $0.04 \mathrm{pA}$ at $2 \mathrm{~V}$ reverse bias with breakdown voltage $\sim 38 \mathrm{~V}$. After wire bonding the devices in parallel, they had a combined dark current of $\sim 0.15 \mathrm{pA}$ at $2 \mathrm{~V}$ reverse bias. A peak quantum efficiency of $38 \%$ was achieved at $440 \mathrm{~nm}$ after

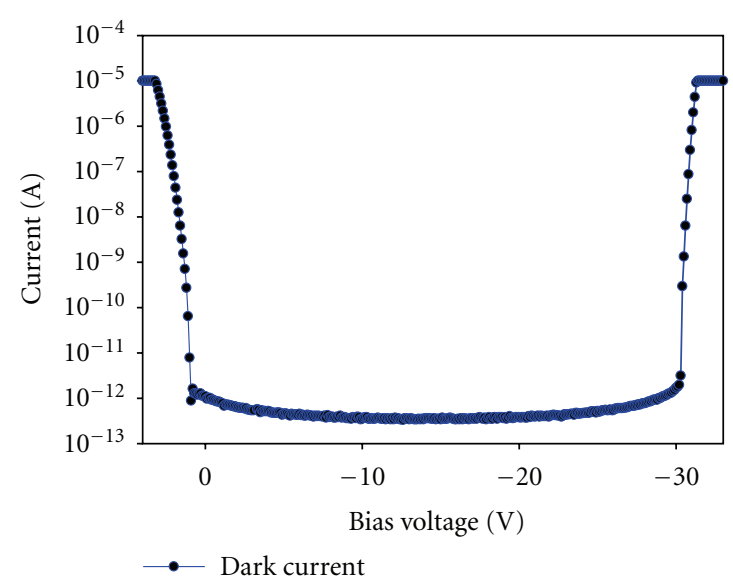

(a)

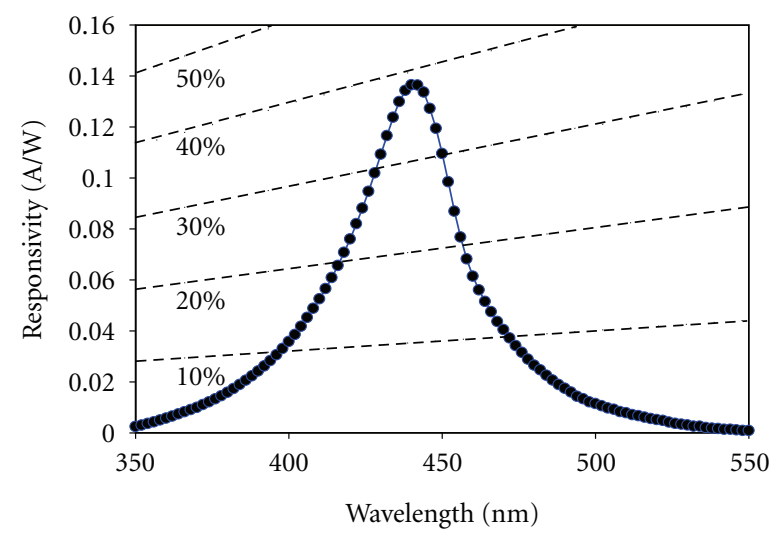

(b)

FIGURE 2: (a) Dark current versus voltage for two devices combined in parallel; (b) quantum efficiency versus wavelength for a single bonded device.

bonding; this reflects a $10 \%$ loss in efficiency due to bonding. Figure 2 shows the device characteristics.

\section{Measurement Setup}

Fused silica capillaries with inner and outer diameters of $320 \mu \mathrm{m}$ and $435 \mu \mathrm{m}$, respectively, were purchased from Polymicro Technologies. A window was opened in the capillary by removing a short strip of the polyimide coating. This window was aligned with the photodiodes, and the capillary was affixed to the detector system, thus integrating the two parts. Forced air was used to push solution from a vial in order to fill the capillary. The whole system was placed on a flat holder with openings for both the capillary and the excitation light. A $266 \mathrm{~nm}$ pulsed laser with $7.3 \mathrm{kHz}$ repetition rate and a $400 \mathrm{ps}$ pulse width was used as the excitation light source. The laser is a compact model and consists of controller box length 6.5 inches, width 5 inches, and height 4 inches as well as the actual laser which is 6 by 3 by 2 inches. The capillary was illuminated by focusing the laser signal with a microscope objective at 90 degrees to the photodiodes. Any light from the laser that is detected 


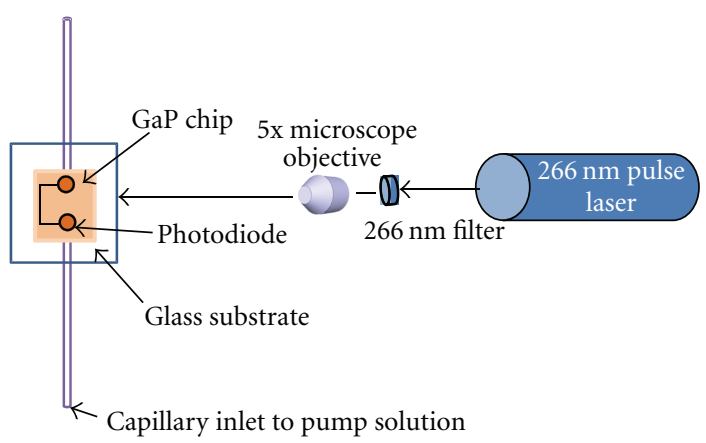

Figure 3: Schematic depiction of measurement setup.

by the photodiodes contributes to the background noise level; to reduce the scattered laser light, a narrow-band filter $266 \pm 10 \mathrm{~nm}$ was placed before the microscope objective. A schematic of the measurement apparatus is depicted in Figure 3.

\section{Linear Fluorescence Detection}

Ciprofloxacin powder was purchased from Fluka, SigmaAldrich, and a stock solution of $100 \mathrm{ppm}$ of ciprofloxacin with equal parts of methanol and deionized water was prepared. The stock solution was further diluted with equal parts of methanol and de-ionized to prepare varying concentrations.

Two GaP photodiodes connected in parallel were used for these measurements. Each device was $220 \mu \mathrm{m}$ in diameter. For linear detection, the devices were biased at $-2 \mathrm{~V}$ and the average current was measured with a Keithley 6430 SourceMeter. The laser was focused to a spot size of $\sim 150 \mu \mathrm{m}$. For light incident perpendicular to the detector $<1 \%$ of the scattered is detected. In operation, light is incident perpendicular to the detector and the scattered light accounts for $<1 \%$ of the signal. When the laser is focused on the capillary directly adjacent to either detector, the scattered light signal in the other detector is below the detection limit. The lowest background current was therefore achieved when the laser was positioned on the capillary adjacent to one of the detectors. Since the fluorescence from the ciprofloxacin is anisotropic, the fluorescence was detected by both photodiodes even though the sample was illuminated only under the first detector. Placing the two detectors in parallel effectively increases the fluorescence detection area while the excitation was proximate to a single device, which significantly reduced the background level.

The photodiode current was measured as ciprofloxacin of different concentrations were pumped through the capillary. When the current reached a stable maximum, a ten data point average was recorded. Methanol was pumped through the capillary after each ciprofloxacin concentration. The methanol flushes any traces on ciprofloxacin from the capillary. This process was continued until the total current returned to the background level. Each concentration was measured three times, and the averaged data along with standard deviation bars is shown in Figure 4. Concentrations

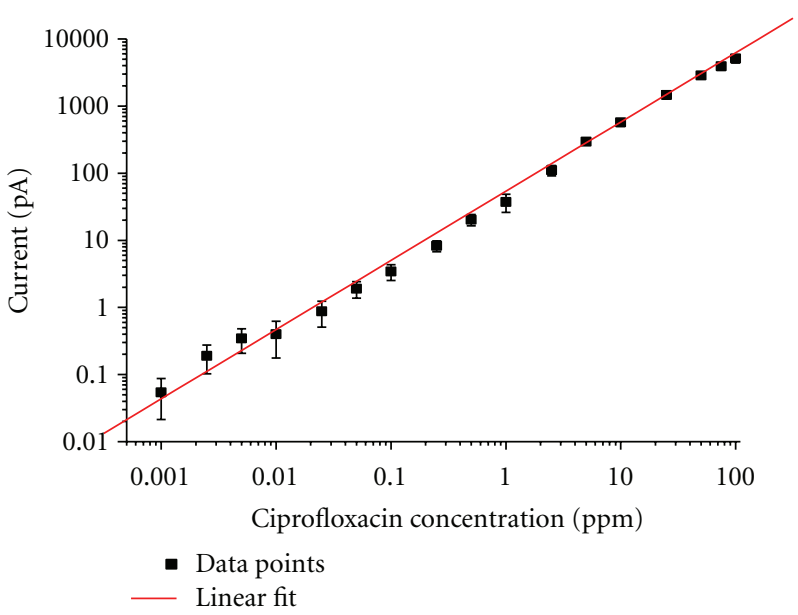

FIGURE 4: Linear detection photocurrent versus ciprofloxacin concentration.

from $1 \mathrm{ppb}$ to $100 \mathrm{ppm}$ were measured. The system showed a linear response for concentrations in this range.

\section{Conclusion}

Integration of two parallel connected photodiodes with a microcapillary was investigated. Individual devices had a peak quantum efficiency of $38 \%$, and the connected parallel devices had a combined dark current of $0.15 \mathrm{pA}$, both at reverse bias $2 \mathrm{~V}$. This system is a simple and effective way of detecting fluorescence from solutions in the wavelength range $400-480 \mathrm{~nm}$. It can potentially be used for initial measurements but, due to its inability to separate analtytes, is not a replacement for HPLC or CE. Linear detection of ciprofloxacin concentrations from $100 \mathrm{ppm}$ down to $1 \mathrm{ppb}$ was achieved.

\section{References}

[1] L. M. Smith, J. Z. Sanders, and R. J. Kaiser, "Fluorescence detection in automated DNA sequence analysis," Nature, vol. 321, no. 6071, pp. 674-679, 1986.

[2] M. L. Chabinyc, D. T. Chiu, J. C. McDonald et al., "An integrated fluorescence detection system in poly(dimethylsiloxane) for microfluidic applications," Analytical Chemistry, vol. 73, no. 18, pp. 4491-4498, 2001.

[3] J. R. Webster, M. A. Burns, D. T. Burke, and C. H. Mastrangelo, "Monolithic capillary electrophoresis device with integrated fluorescence detector," Analytical Chemistry, vol. 73, no. 7, pp. 1622-1626, 2001.

[4] R. A. Mathies, T. Kamei, B. M. Paegel, J. R. Scherer, A. M. Skelley, and R. A. Street, "Integrated hydrogenated amorphous Si photodiode detector for microfluidic bioanalytical devices," Analytical Chemistry, vol. 75, no. 20, pp. 5300-5305, 2003.

[5] R. M. Hoffman, "The multiple uses of fluorescent proteins to visualize cancer in vivo," Nature Reviews Cancer, vol. 5, no. 10, pp. 796-806, 2005.

[6] M. K. Araz, C. H. Lee, and A. Lal, "Ultrasonic separation in microfluidic capillaries," in Proceedings of the 2003 IEEE Ultrasonics Symposium, pp. 1111-1114, October 2003. 
[7] S. Jeonggi and L. P. Lee, "Fluorescence amplification by selfaligned integrated microfluidic optical systems," in Proceedings of the 12th International Conference in TRANSDUCERS, SolidState Sensors, Actuators and Microsystems, vol. 2, pp. 1136$1139,2003$.

[8] C. J. Easley, J. M. Karlinsey, J. M. Bienvenue et al., "A fully integrated microfluidic genetic analysis system with samplein-answer-out capability," Proceedings of the National Academy of Sciences of the United States of America, vol. 103, no. 51, pp. 19272-19277, 2006.

[9] P. K. Wong, Y. K. Lee, and C. M. Ho, "Deformation of DNA molecules by hydrodynamic focusing," Journal of Fluid Mechanics, no. 497, pp. 55-65, 2003.

[10] L. Kaartinen, M. Salonen, L. Alli, and S. Pyorala, "Pharmacokinetics of enrofloxacin after single intravenous, intramuscular and subcutaneous injections in lactating cows," Journal of Veterinary Pharmacology and Therapeutics, vol. 18, no. 5, pp. 357-362, 1995.

[11] O. R. Idowu and J. O. Peggins, "Simple, rapid determination of enrofloxacin and ciprofloxacin in bovine milk and plasma by high-performance liquid chromatography with fluorescence detection," Journal of Pharmaceutical and Biomedical Analysis, vol. 35, no. 1, pp. 143-153, 2004.

[12] X. Zhou, D. Xing, D. Zhu, Y. Tang, and L. Jia, "Development and application of a capillary electrophoresiselectrochemiluminescent method for the analysis of enrofloxacin and its metabolite ciprofloxacin in milk," Talanta, vol. 75, no. 5, pp. 1300-1306, 2008.

[13] O. Ballesteros, I. Toro, V. Sanz-Nebot, A. Navalón, J. L. Vílchez, and J. Barbosa, "Determination of fluoroquinolones in human urine by liquid chromatography coupled to pneumatically assisted electrospray ionization mass spectrometry," Journal of Chromatography B, vol. 798, no. 1, pp. 137-144, 2003.

[14] K. H. Bannefeld, H. Stass, and G. Blaschke, "Capillary electrophoresis with laser-induced fluorescence detection, an adequate alternative to high-performance liquid chromatography, for the determination of ciprofloxacin and its metabolite desethyleneciprofloxacin in human plasma," Journal of Chromatography B, vol. 692, no. 2, pp. 453-459, 1997.

[15] G. A. Wilson and J. Brady, "Design considerations and signal processing algorithms for laser-induced fluorescence airborne pathogen sensors," in Proceedings of the International Society for Optical Engineering, (SPIE 5617), pp. 1-13, London, UK, 2004.

[16] J. Campbell, D. McIntosh, Q. Z. F. J. Lara, and J. Landers, "Flip-chip bonded GaP photodiodes for detection of 400 480 nm fluorescence," Photonics Technology Letters, vol. 23, no. 13, pp. 878-880, 2011.

[17] A. L. Beck, B. Yang, S. Wang et al., "Quasi-direct UV/blue GaP avalanche photodetectors," IEEE Journal of Quantum Electronics, vol. 40, no. 12, pp. 1695-1699, 2004. 

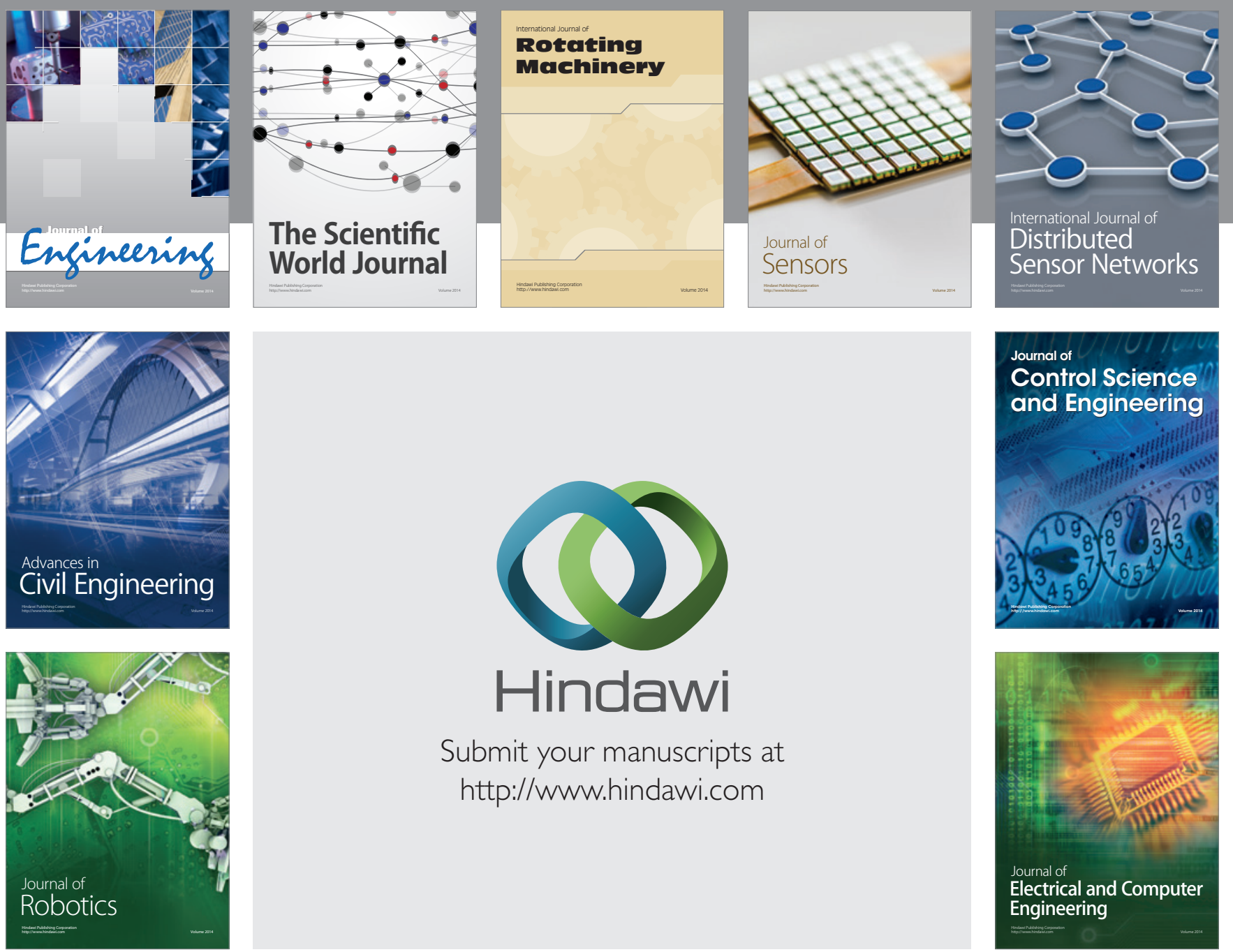

Submit your manuscripts at

http://www.hindawi.com
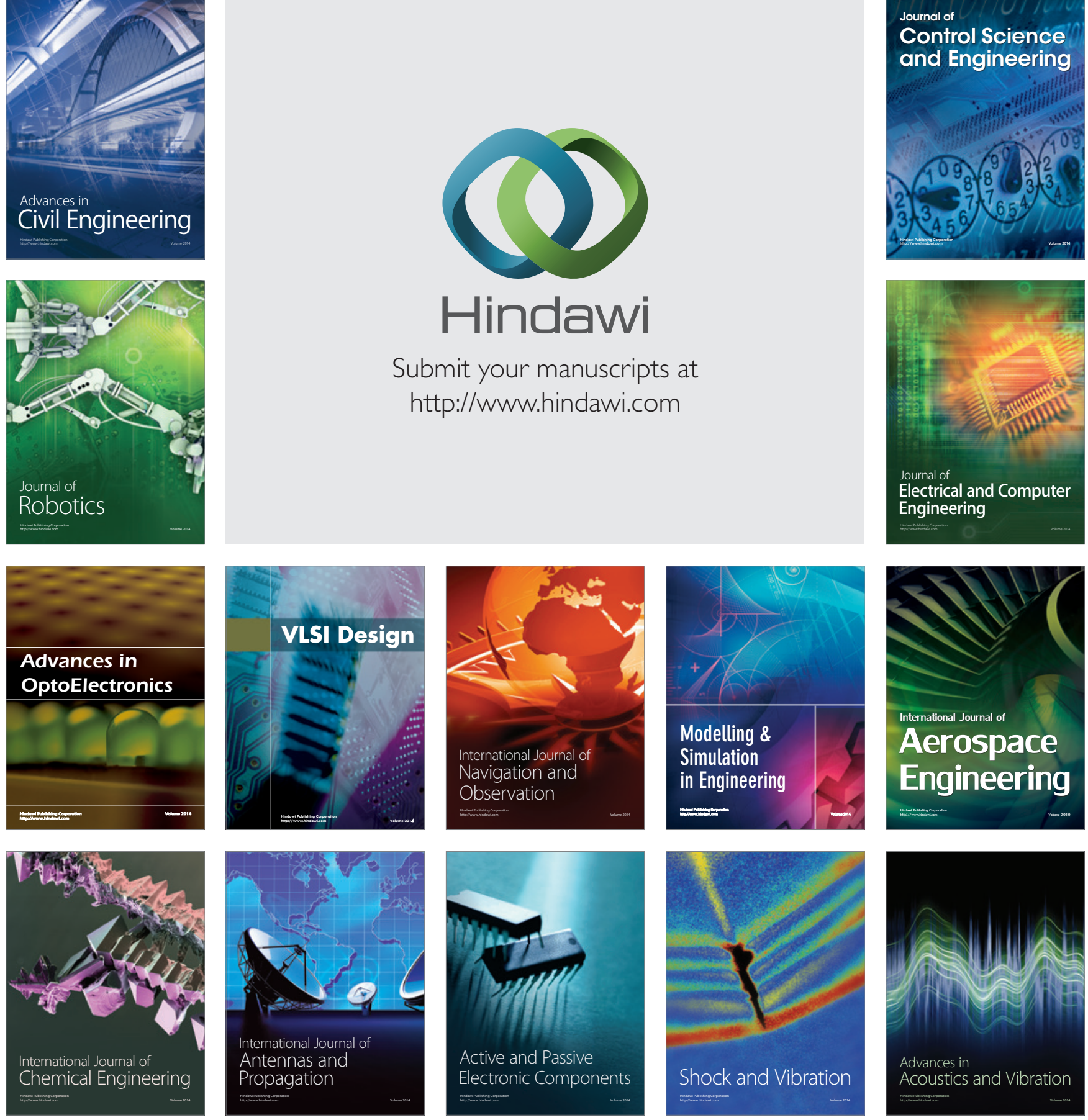